\title{
Noise-aware Evolutionary TDMA Optimization for Neuronal Signaling in Medical Sensor-Actuator Networks
}

\author{
Junichi Suzuki \\ Department of Computer Science \\ University of Massachusetts, Boston \\ Boston, MA, 02125-3393, USA \\ jxs@cs.umb.edu
}

\author{
Pruet Boonma \\ Department of Computer Engineering \\ Chiang Mai University \\ Chiang Mai, 50200, Thailand \\ pruet@eng.cmu.ac.th
}

\begin{abstract}
Neuronal signaling is one of several approaches to network nanomachines in the human body. This paper formulates a noisy optimization problem for a neuronal signaling protocol based on Time Division Multiple Access (TDMA) and solves the problem with a noise-aware optimizer that leverages an evolutionary algorithm. The proposed optimizer is intended to minimize signaling latency by multiplexing and parallelizing signal transmissions in a given neuronal network, while maximizing signaling robustness (i.e., unlikeliness of signal interference). Since latency and robustness objectives conflict with each other, the proposed optimizer seeks the optimal trade-offs between them. It exploits a nonparametric (i.e.. distribution-free) statistical operator because it is not fully known what distribution(s) noise follows in each step/component in neuronal signaling. Simulation results show that the proposed optimizer efficiently obtains quality TDMA signaling schedules and operates a TDMA protocol by balancing conflicting objectives in noisy environments.
\end{abstract}

\section{Categories and Subject Descriptors}

C.2.2 [Computer-Communication Networks]: Network Protocols; C.3 [Special-purpose and Application-based Systems]: Signal processing systems; I.2.8 [Artificial Intelligence]: Problem Solving, Control Methods, and SearchHeuristic methods

\section{Keywords}

Molecular communication, Neuronal networks, TDMA scheduling, Evolutionary multiobjective optimization algorithms

\section{INTRODUCTION}

A nanoscale system consists of one or more nanomachines, which are nanoscale devices that perform simple computation, sensing and/or actuation tasks [17, 25]. Nanomachines may be man-made devices built in the top-down approach, downscaling the current microelectronic and microelectromechanical technologies, or in the bottom-up approach, assembling synthesized nanomaterials such as graphene nanoribbons and carbon nanotubes. Alternatively, nanomachines

Permission to make digital or hard copies of all or part of this work for personal or classroom use is granted without fee provided that copies are not made or distributed for profit or commercial advantage and that copies bear this notice and the full citation on the first page. Copyrights for components of this work owned by others than ACM must be honored. Abstracting with credit is permitted. To copy otherwise, or republish, to post on servers or to redistribute to lists, requires prior specific permission and/or a fee. Request permissions from permissions@acm.org.

GECCO'14, July 12-16, 2014, Vancouver, BC, Canada.

Copyright 2014 ACM 978-1-4503-2881-4/14/07 ...\$15.00.

http://dx.doi.org/10.1145/2598394.2609854. may be bio-hybrid, integrating man-made nanostructures with biological materials such as DNA strands and genetically engineered cells, or bio-enabled, synthesizing biological materials without man-made nanostructures [9,21].

An emerging design strategy for nanoscale systems is to network nanomachines for operating in larger physical spaces in higher spatial and temporal resolutions. Although individual nanomachines are limited in computation, sensing and actuation capabilities, an assembly of nanomachines can potentially organize into a "large-scale" network that spreads on centimeter to meter scale and collaboratively performs tasks that no individual nanomachines could.

Molecular communication is one of a few options to network nanomachines. It utilizes molecules as a communication medium between nanomachines $[17,25]$. Due to its advantages such as inherent nanometer scale, biocompatibility and energy efficiency [9], a key application domain of molecular communication is intrabody nanonetworks where nanomachines are networked to perform sensing and actuation tasks for biomedical and prosthetic purposes. Those tasks include in-situ physiological sensing, biomedical anomaly detection, targeted drug delivery, medical operations with cellular/molecular level precision, neural signal transduction and neuromuscular implant control.

This paper focuses on molecular communication that utilizes neurons as a primary component to build intrabody nanonetworks. A neuron-based intrabody nanonetwork consists of a set of nanoscale sensor/actuator nodes and a network of neurons that are artificially formed into a particular topology It allows individual nodes to interface with neurons and communicate to other nodes with neuronal (i.e., electrochemical) signals through a chain of neurons.

This paper formulates a noisy multiobjective optimization problem for a neuronal signaling protocol, called Neuronal TDMA, and approaches the problem with a noise-aware evolutionary multiobjective optimization algorithm (EMOA). Neuronal TDMA performs single-bit Time Division Multiple Access (TDMA) scheduling for individual nodes to activate their nearby neurons and multiplex/parallelize signal transmissions in a given neuronal network.

The proposed EMOA heuristically seeks the optimal TDMA schedules (i.e., which neurons to activate and when to activate them to trigger signal transmissions) for nodes by evolving a set of solution candidates (or individuals) toward the optima through generations. Each individual represents a particular TDMA schedule for individual nodes. The proposed EMOA is designed to address two important issues in neuronal signaling: interference and noise. When differ- 


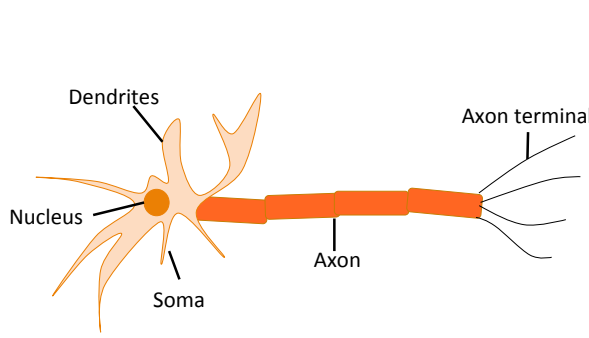

Figure 1: Structure of a neuron

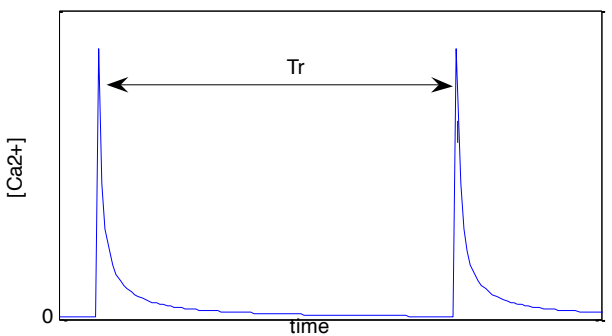

Figure 2: Intracellular $\mathrm{Ca}^{2+}$ concentration

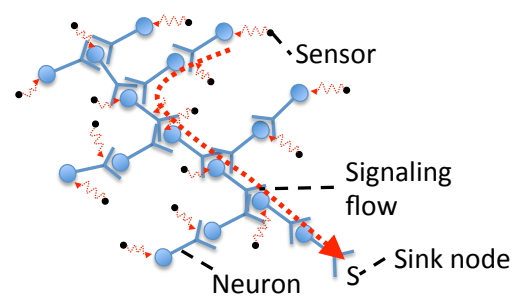

Figure 3: An Example SensorActuator Network ent signals attempt to travel through a neuron simultaneously, they interfere (or collide) with each other [3]. This leads to the loss or corruption of information encoded with the signals. The proposed EMOA is designed to avoid signal interference to ensure that signals reach the destination while multiplexing/parallelizing signal transmissions to improve performance measures such as signaling latency.

Neuronal signaling is inherently noisy [6]. For example, thermal, current and chemical noise affects the latency of signal transmission. Due to noise, the same individual (i.e., TDMA schedule) can yield different performance measures from time to time. This degenerates individual-to-individual comparison; for example, the proposed EMOA may mistakenly judge that an inferior individual outperforms a superior one. More importantly, noise can cause signal interference unexpectedly. Even if the proposed EMOA determines a feasible individual, which is designed to perform interferencefree signaling, the individual may actually become infeasible, causing signal interference, due to noise.

The proposed EMOA performs a statistical noise-aware operator for individual-to-individual comparison. (Individualto-individual comparison operators are often called dominance operators in the area of EMOAs.) Using the noiseaware operator, called the $\alpha$-dominance, the proposed EMOA is designed to seek the Pareto-optimal individuals (TDMA schedules) with respect to conflicting optimization objectives: signaling latency and signaling robustness (unlikeliness of signal interference). Simulation results show that the proposed EMOA successfully allows Neuronal TDMA to perform signal transmissions in noisy environments and reveals the trade-offs between signaling latency and robustness. It is also verified that the $\alpha$-dominance operator outperforms existing noise-aware operators.

\section{RELATED WORK}

Compared to other molecular communication approaches (e.g., molecular motors, calcium signaling and bacteria communication), neuron-based communication has such advantages as long distance coverage, high speed signaling (up to $90 \mathrm{~m} / \mathrm{s}$ ) and low attenuation in signaling [13].

Balasubramaniam et al. first examined a TDMA protocol for neuronal networks [1]. Neuronal TDMA extended it with an EMOA that considers communication performance objectives such as signaling yield and latency [26]. This paper extends [26] by considering communication robustness as well as communication performance in noisy environments.

In the area of EMOAs, there exist various existing work to handle noise/uncertainties in objective functions. Most of them take parametric approaches, which assume particular noise distributions in advance; for example, normal distributions [8], uniform distributions [27] and Poisson distributions [28]. Given a noise distribution, existing noise-aware dominance operators collect objective value samples from each individual in order to determine superior-inferior relationships among individuals. In contrast, the $\alpha$-dominance operator assumes no noise distributions in advance because it is not fully known what distribution noise follows in each step/component in the entire neuronal signaling process.

\section{BACKGROUND}

This section provides preliminaries on neuronal signaling and neuron-based intrabody sensor-actuator networks.

\subsection{Neuronal Signaling}

Neurons are a fundamental component of the nervous system, which includes the brain and the spinal cord. They are electrically excitable cells that process and transmit information via electrical and chemical signaling.

A neuron consists of cell body (or soma), dendrites and axon (Figure 1) [3]. A soma varies from 4 to 100 micrometers in diameter. Dendrites are thin structures that arise from the soma. The length of a dendrite is up to a few hundred micrometers. Dendrites receive the majority of inputs to a neuron. An axon is a cellular extension that arises from the soma. It travels through the body in bundles called nerves. Its length can be over one meter in the human nerve that arises from the spinal cord to a toe.

Neurons are connected with each other to form a network(s). They communicate via synapses, each of which is a junction between two neurons. A synapse contains molecular machinery that allows a (presynaptic) neuron to transmit a chemical signal to another (postsynaptic) neuron. Signals are transmitted from the axon of a presynaptic neuron to a dendrite of a postesynaptic neuron. An axon transmits an output signal to a postsynaptic neuron, and a dendrite receives an input signal from a presynaptic neuron.

Presynaptic and postsynaptic neurons maintain voltage gradients across their membranes by means of voltage-gated ion channels, which are embedded in the presynaptic membrane to unbalance intracellular and extracellular concentration of ions (e.g., $\mathrm{Ca}^{2+}$ ) [24]. Changes in the crossmembrane ion concentration (i.e., voltage) can alter the function of ion channels. If the concentration changes by a large enough amount (e.g., approx. $80 \mathrm{mV}$ in a giant squid), ion channels start pumping extracellular ions inward. Upon the increase in intracellular ion concentration, the presynaptic neuron releases a chemical called a neurotransmitter (e.g., acetylcholine), which travels through the synapse from 
the presynaptic postsynaptic neuron. The neurotransmitter electrically excites the postsynaptic neuron, which in turn generates an electrical pulse called an action potential. This signal travels rapidly along the neuron's axon and activates synaptic connections (i.e., opens ion channels) when it arrives at the axon's terminals. This way, an action potential triggers cascading neuron-to-neuron communication.

Figure 2 shows how $\mathrm{Ca}^{2+}$ concentration changes in a neuron. When the concentration peaks, the neuron releases a neurotransmitter(s) and goes into a refractory period $\left(T_{r}\right.$ in Figure 2), which is the time required for the neuron to replenish its internal $\mathrm{Ca}^{2+}$ store. During $T_{r}$, it cannot process any incoming signals. The refractory period is approximately two milliseconds in a giant squid.

\subsection{Neuron-based Intrabody Nanonetworks}

This paper assumes neuronal signaling in a three-dimensional network of natural neurons that are artificially grown and formed into particular topology patterns. This assumption is made upon numerous research efforts to grow neurons on substrates [18] and form topologically-specific neuronal networks $[14,16,20,29]$.

Figure 3 illustrates a schematic neuron-based intrabody nanonetwork. It contains an artificially-grown neuronal network and several nanomachines such as sensors and a sink. Sensors utilize neuronal signaling to deliver sensor data to a sink. As a potential application, sensors may periodically monitor certain physiological status and report physiological data or biomedical anomalies to the sink. The sink may work as a transducer that converts incoming electrochemical signals to electrostatic or electromagnetic signals. Electrostatic signals may carry sensor data to an on-body (i.e., epidermal) device(s) through a body-coupled communication scheme [19,31]. Electromagnetic signals may carry sensor data to an around-body device(s) such as a smartphone and tablet computer.

This paper assumes that nanomachines (e.g., sensor and sink nodes) interact with neuronal networks in a non-invasive manner. This means that it is not required to insert particular materials (e.g., carbon nanotubes) into neurons so that nanomachines can trigger and receive signals. For example, nanomachines may use chemical agents (e.g., acetylcholine and mecamylamine [1]) or light $[11,12]$.

Other potential applications are neurointerfaces that leverage in-situ sensing and actuation for prosthetic devices; for example, neuroprosthetic bladder control (Figure 4). In a normal bladder, a sensory nerve senses that the bladder is full of urine and transmits a sensory signal (i.e., the bladder's sense of fullness) to the brain. Whenever appropriate, the brain sends out a control signal through the spinal cord to contract the bladder, relax the sphincter and trigger urination. However, nervous system disorders (e.g., spinal cord injuries and subsequent paralysis) can disrupt those signals to/from the brain and eliminate the fullness sensation and muscle control. A person with this kind of disorder is forced to empty his/her bladder with a catheter.

In-situ sensing and actuation can help correct incontinence. In Figure 4, a particular portion of a spinal nerve, called dorsal root ganglion, is teased out and interfaced to sensor nodes. The sensors intercept neuronal signals from the nerve and forwards them to the sink node. The sink may determine whether the bladder is full, and if it is full, transmits electrostatic or electromagnetic signals to an on/around-body node(s), which in turn notify the bladder's fullness to the patient. A neurostimulator(s) connected to the nerve issues high-frequency stimulation to prevent the bladder from emptying itself. Whenever ready to urinate, the patient uses his/her on/around-body node to issue an electrostatic or electromagnetic signal to a subepidermal node, which in turn transduces the signal to a neuronal electrochemical signal and transmits it to in-situ a neurostimulator(s). Each neurostimulator delivers low-frequency stimulation or stop stimulation so that the bladder to empty. This intervention can be a less invasive alternative to the current state of the art in neuroprosthetic bladder control (e.g., [4]).

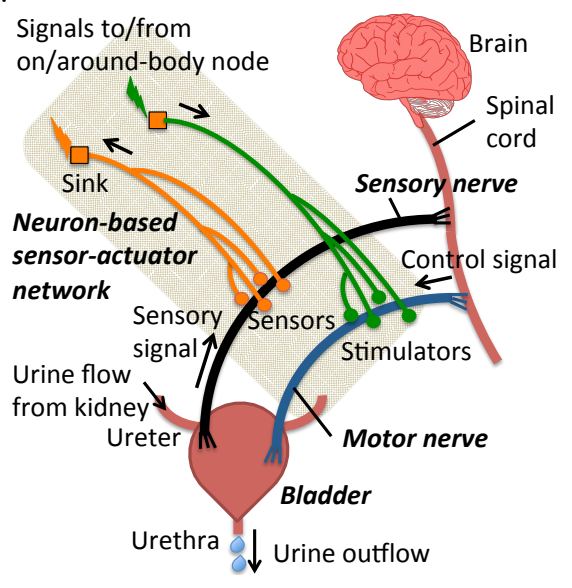

Figure 4: Neuroprosthetic Bladder Control

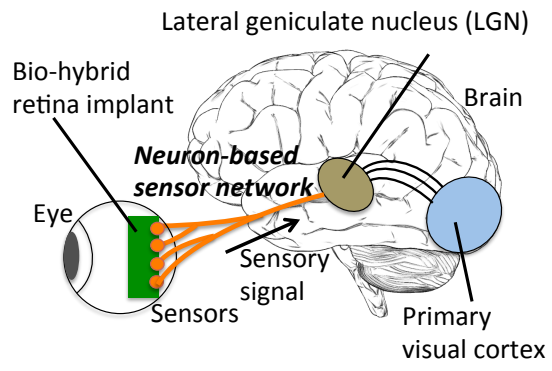

Figure 5: Neuroprosthetic Visual Pathway

Another potential neurointerface application is a bio-hybrid visual prosthesis. In a normal retina, photoreceptor cells perform phototransduction, which converts light (visible electromagnetic radiation) to electrical signals in retinal ganglion cells. Ganglion cells are located near the inner surface of the retina, and their axons extend to the brain. Those axons form the optic nerve. Ganglion cells receive visual information from photoreceptor cells by means of electrical signals and transmits it to the lateral geniculate nucleus of the thalamus, which in turn projects to the primary visual cortex in the occipital lobe.

One of treatment strategies for retinal disorders is to use retinal implants. For example, an implant proposed in [22] bypasses photoreceptor cells and stimulates the optic nerve (i.e., ganglion cells) directly with signals from a phototransduction device that is equipped with a video camera.

An emerging design approach is to bypasses the optic nerve as well as photoreceptor cells [30]. A neuron-based nanonetwork can replace the optic nerve and connect an implant with the lateral geniculate nucleus as a transplanted 
visual pathway (Figure 5). The implant contains nanomachines that receive signals (visual information) from a phototransduction device through a wired or wireless means. Cultured neurons are attached onto those nanomachines, and their axons are guided to the lateral geniculate nucleus. This bio-hybrid implant would be useful for patients with diseases in the optic nerve such as glaucoma and diabetic retinopathy.

\section{NOISY OPTIMIZATION PROBLEM IN NEURONAL TDMA}

Neuronal TDMA performs a single-bit TDMA communication that periodically assigns a time slot to each sensor. Sensors activate (or fire) neurons, one after the other, each using its own time slot. This allows multiple sensors to transmit signals to the sink through the shared neuronal network. Each sensor transmits a single-bit within a single time slot. This single-bit-per-slot design is based on two assumptions: (1) a signal (i.e., action potential) is interpreted with two levels of amplitudes, which represent 0 and 1, and (2) after a signal transmission, a neuron goes into a refractory period $\left(T_{r}\right.$ in Figure 2$)$.

As described in Section 1, an important goal of Neuronal TDMA is to avoid signal interference, which occurs when multiple signals fire the same neuron at the same time and leads to corruption of transmitted sensor data at the sink. Signals can easily interfere with each other if sensors fire their neighboring neurons randomly. Neuronal TDMA is intended to eliminate signal interference by scheduling which sensors fire which neurons with respect to time. The proposed EMOA seeks the optimal TDMA schedules for a set of sensors in a given neuronal network.

Figure 6 shows an example neuronal network that has five neurons $\left(n_{1}\right.$ to $\left.n_{5}\right)$ and four nodes (three sensors, $s_{1}, s_{2}$ and $s_{3}$, and a sink). Figure 7 illustrates an example TDMA schedule for those sensors to fire neurons. The scheduling cycle period lasts five time slots $\left(T_{s}=5\right)$. The sensor $s_{1}$ fires the neuron $n_{4}$ to initiate signaling in the first time slot $T_{1}$. The signal travels through $n_{5}$ in the next time slot $T_{2}$ to reach the sink. $s_{2}$ transmits a signal on $n_{3}$ in $T_{2}$. During $T_{2}$, two signals travel in the neuronal network in parallel. The duration of each time slot ( $T_{u}$ in Figure 7$)$ is designed as the sum of three time periods: (1) synaptic delay, which is the time for neurotransmitters to travel through a synapse from a presynaptic neuron or a sensor and generate an action potential in a postsynaptic neuron, (2) intracellular transmission delay, which is the time for an action potential to travel within a neuron (i.e., from its dendrite terminal to its axon terminal) and (3) a refractory period.

\subsection{Optimization Constraints and Objectives}

The scheduling problem this paper focuses on is defined as an optimization problem where a neuron-based nanonetwork contains $M$ sensors, $S=\left\{s_{1}, s_{2}, \ldots, s_{i}, \ldots, s_{M}\right\}$, and $N$ neurons, $N=\left\{n_{1}, n_{2}, \ldots, n_{j}, \ldots, n_{N}\right\}$.

The proposed EMOA is designed to meet two optimization constraints. The first constraint enforces that at most one signal can pass through each neuron in a single time slot. (Otherwise, signal interference occurs.) The second constraint enforces each sensor transmits one signal during the scheduling cycle $T_{s}$ A TDMA schedule is said to be feasible if it never violates constraints. On the contrary, it is said to be infeasible if it violates any of constraints. An example TDMA schedule in Figure 7 is feasible.

The proposed EMOA considers two optimization objectives: signaling latency and signaling robustness. Signaling latency $\left(f_{L}\right)$ is computed with Equation 1. It indicates how soon the sink receives all signals from all $M$ sensors. It is to be minimized.

$$
f_{L}=\max _{s_{i} \in S} t_{a}^{s_{i}}
$$

$t_{a}^{s_{i}}$ denotes the arrival time at which the sink receives a signal that $s_{i}$ transmits. $f_{L}$ determines the scheduling cycle period $T_{s}\left(T_{s}=f_{L}\right)$. In Figure $7, f_{L}=5$.

Signlaing robustness $\left(f_{R}\right)$ indicates the unlikeliness for signals to interfere with each other on shared neurons $N^{\prime}=$ $\left\{n_{1}^{\prime}, n_{2}^{\prime}, \ldots, n_{h}^{\prime}, \ldots, n_{H}^{\prime}\right\} \subset N$. Shared neurons are the neurons that sensors share to transmit their signals to the sink. In Figure $6, n_{2}$ and $n_{5}$ are shared neurons. ( $s_{2}$ and $s_{3}$ share $n_{2} . s_{1}, s_{2}$ and $s_{3}$ share $n_{5}$.) Higher robustness means lower chances of signal interference. $f_{R}$ is computed with Equation 2. It is to be maximized.

$$
f_{R}=\sum_{l=1}^{M} \sum_{m=1}^{M} \sum_{h=1}^{H} \frac{I_{s}}{T_{u}}\left(t_{a}^{n_{h}^{\prime}\left(s_{l}\right)}-t_{a}^{n_{h}^{\prime}\left(s_{m}\right)}\right), \quad l \neq m
$$

$t_{a}^{n_{h}^{\prime}\left(s_{l}\right)}$ denotes the arrival time at which a signal from the $l$-th sensor $s_{l}$ arrives at the $h$-th shared neuron $n_{h}^{\prime} \cdot t_{a}^{n_{h}^{\prime}\left(s_{l}\right)}-$ $t_{a}^{n_{h}^{\prime}\left(s_{m}\right)}=0$ if signals from $s_{l}$ and $s_{m}$ interfere on $n_{h}^{\prime}$. In Figure 7, $s_{2}$ 's and $s_{3}$ 's signals pass through $n_{2}$ in $T_{3}$ and $T_{4}$, respectively. Thus, $t_{a}^{n_{2}\left(s_{3}\right)}-t_{a}^{n_{2}\left(s_{2}\right)}=T_{u}$. Note that $I_{s}=1$ if $t_{a}^{n_{h}^{\prime}\left(s_{l}\right)}>t_{a}^{n_{h}^{\prime}\left(s_{m}\right)}$; otherwise, $I_{s}=0$.

\subsection{Impacts of Noise on Objective Functions}

As described in Section 1, this paper considers noise that varies the speed and latency of signal transmissions. Major contributors to noise are variances of synaptic delay and refractory period.

Noise impacts both objective functions (Equations 1 to 2). It impacts $f_{F}, f_{L}$ and $f_{R}$ by varying $t_{a}$ in Equations 1 and 2 . Therefore, they are redefined as follows for signaling in noisy environments.

$$
\begin{aligned}
& f_{L}^{e}=f_{L}+e_{L} \\
& f_{R}^{e}=f_{R}+e_{R}
\end{aligned}
$$

This way, noise-aware objectives are defined with additive noise $\left(e_{L}\right.$ and $\left.e_{R}\right)$, which can be positive and negative.

As $t_{a}$ varies, signal interference can occur even if the onesignal-per-slot constraint (see Section 4.1) is satisfied. In Figure $7, s_{2}$ 's signal travels on $n_{2}$ during $T_{3}$, and $s_{3}$ 's signal travels on $n_{2}$ during $T_{4}$. This signaling schedule satisfies the one-signal-per-slot constraint; however, the two signals can interfere if $s_{2}$ 's signal arrives at $n_{2}$ sometime in $T_{4}$ and/or if $s_{3}$ 's signal arrives at $n_{2}$ sometime in $T_{3}$ due to noise. This means that noise can degenerate a feasible individual (i.e., signaling schedule) to be infeasible.

\section{THE PROPOSED EMOA}

The proposed EMOA is designed to solve the noisy optimization problem described in Section 4. It iteratively 

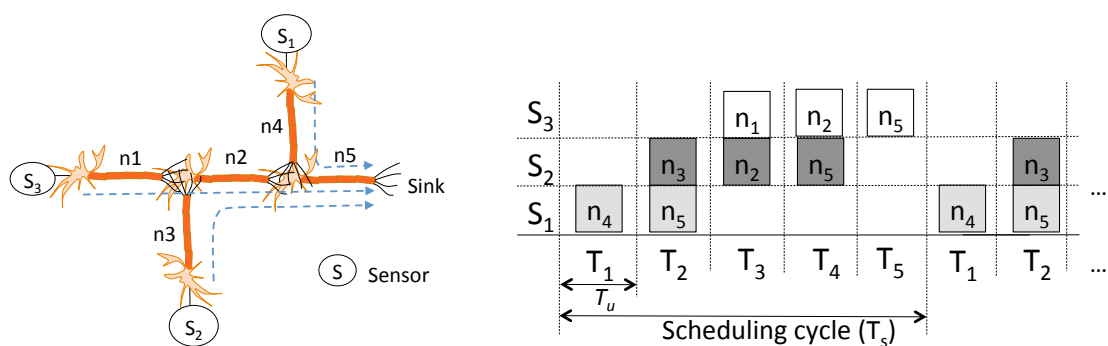

\section{Figure 6: An Example Neu- Figure 7: An Example TDMA Schedule Figure 8: An Example In- ronal Network \\ dividual}

\begin{tabular}{|c|c|c|c|c|c|}
\hline $\mathrm{S}_{3}$ & 0 & 0 & 1 & 0 & 0 \\
\hline $\mathrm{S}_{2}$ & 0 & 1 & 0 & 0 & 0 \\
\hline $\mathrm{S}_{1}$ & 1 & 0 & 0 & 0 & 0 \\
\hline
\end{tabular}

evolves the population of individuals (i.e., TDMA schedules) via several operators (e.g., crossover, mutation and selection operators) toward the Pareto optima.

Each individual represents a particular TDMA schedule for $M$ sensors. Figure 8 shows the structure of an example individual, which encodes the TDMA schedule in Figure 7. In this example, $s_{1}$ fires its neighboring neuron, $n_{4}$, in the first time slot $T_{1} . s 2$ and $s_{3}$ fire $n_{2}$ and $n_{1}$ in $T_{2}$ and $T_{3}$, respectively.

\subsection{The $\alpha$-Dominance Operator}

This section describes the notion of $\alpha$-dominance. It determines a statistical superior-inferior relationship, called the $\alpha$-dominance relationship, between given two individuals using their objective value samples. It extends a classical dominance relationship [23], in which an individual $i$ is said to dominate an individual $j$ (denoted by $i \succ j$ ) if the both of the following conditions are hold:

- $i$ 's objective values are superior than, or equal to, $j$ 's in all objectives.

- $i$ 's objective values are superior than $j$ 's in at least one objectives.

The $\alpha$-dominance operator takes objective value samples of given two individuals, estimates the impacts of noise on the samples, and determines whether it is confident enough to judge which one is superior/inferior between the two individuals. The operator determines that individual $A \alpha$ dominate individual $B$ (denoted by $A \succ_{\alpha} B$ ), iif:

- $A$ 's and B's objective value samples are classifiable with a statistical confidence level of $\alpha$, and

- $\mathcal{C}(A, B)=1 \wedge \mathcal{C}(B, A)<1$.

In order to examine the first condition, the $\alpha$-dominance operator classifies $A$ 's and $B$ 's objective value samples with Support Vector Machine (SVM), and measures a classification error. (See Step 1 in an example shown in Figure 9.) The error $(e)$ is computed as the ratio of the number of miss-classified samples to the total number of samples. For evaluating the confidence level $(\alpha)$ in a classification error, the $\alpha$-dominance operator computes the classification error's confidence interval $\left(e_{i n t}\right)$ :

$$
e_{i n t}=e \pm t_{\alpha, n-1} \sigma
$$

$t_{\alpha, n-1}$ denotes a single-tail $t$-distribution with $\alpha$ confidence level and $n-1$ degrees of freedom. $n$ denotes the total number of samples. $\sigma$ is the standard deviation of $e$. It is approximated as follows.

$$
\sigma \cong \sqrt{\frac{e}{n}}
$$

If $e_{i n t}$ is significant (i.e., if $e_{\text {int }}$ does not span zero), the $\alpha$-dominance operator cannot classify $A$ 's and $B$ 's samples with the confidence level of $\alpha$. Thus, the operator determines that A and B do not $\alpha$-dominate each other. (See Step 2 in Figure 9.)

If $e_{i n t}$ is not significant (i.e., if $e_{i n t}$ spans zero), the $\alpha$ dominance operator can classify $A$ 's and $B$ 's samples with the confidence level of $\alpha$. Thus, the operator examine the aforementioned second condition. (See Step 2 in an example shown in Figure 9.) It measures $\mathcal{C}$-metric [33] with a classical notion of dominance $(\succ)$ described earlier. $\mathcal{C}(A, B)$ denotes the fraction of individual $B$ 's samples that at least one sample of individual $A$ dominates:

$$
\mathcal{C}(A, B)=\frac{|\{b \in B \mid \exists a \in A: a \succ b\}|}{|B|}
$$

If $\mathcal{C}(A, B)=1$, all of $B$ 's samples are dominated by at least one sample of $A$. If $\mathcal{C}(B, A)<1$, not all of $A$ 's samples are dominated by at least one sample of $B$. The $\alpha$ dominance operator determines $A \succ_{\alpha} B$ if $\mathcal{C}(A, B)=1$ and $\mathcal{C}(B, A)<1$. If $\mathcal{C}(A, B)<1$ and $\mathcal{C}(B, A)<1$, the operator determines neither $A \succ_{\alpha} B$ nor $B \succ_{\alpha} A$. See Figure 9 as well.

Figure 9 shows an example to determine the $\alpha$-dominance relationship between two individuals, $A$ and $B$, with two objectives, $f_{1}$ and $f_{2}$, to be minimized. Individual $A$ and $B$ have seven samples each. First, the $\alpha$-dominance operator classifies these 14 samples in the objective space with SVM and computes $e_{i n t}$. Suppose SVM produces a classification vector as shown in Figure 9. Two samples of $B$ are missclassified; $e=\frac{2}{14}$ (0.143). Thus, $\sigma \cong \sqrt{\frac{0.143}{14}}=0.1$. Assuming the confidence level $\alpha$ of $95 \%, e_{\text {int }}=0.143 \pm 1.771 * 0.1=$ $0.143 \pm 0.1771$. Since $e_{i n t}$ spans zero, $A$ 's and $B$ 's samples are classifiable with the confidence level of $95 \%$. This means that the aforementioned first condition is hold. In order to examine the second condition, the $\alpha$-dominance operator measures $\mathcal{C}(A, B)$ and $\mathcal{C}(B, A)$. In Figure $9, \mathcal{C}(A, B)=1$ and $\mathcal{C}(B, A)=2 / 14<1$. This means that the second condition 


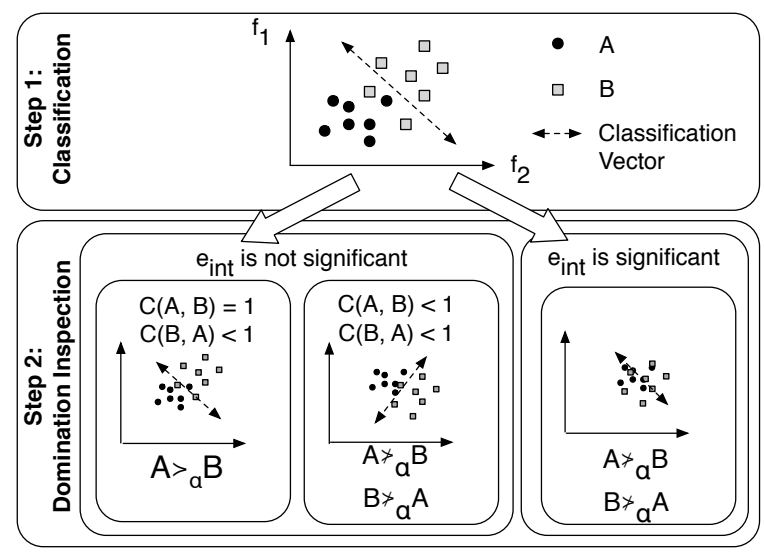

Figure 9: An Example Process to determine the $\alpha$ Dominance Relationship between two individuals (A and $B$ )

is hold. As a re result, the $\alpha$-dominance operator concludes $A \succ_{\alpha} B$.

\subsection{Evolutionary Optimization Process}

Algorithm 1 shows the algorithmic structure of the proposed $\alpha$-dominance based EMOA. At the 0 -th generation, $P$ feasible individuals are randomly generated as the initial population $\mathcal{P}_{0}$ (Line 2 ).

In each generation $(g)$, two parent individuals $\left(p_{1}\right.$ and $\left.p_{2}\right)$ are selected from the current population $\mathcal{P}_{g}$ with binary tournaments (Lines 6 and 7). A binary tournament randomly takes two individuals from $\mathcal{P}_{g}$, compares them based on the notion of $\alpha$-dominance, and chooses a superior one as a parent. With the crossover rate $P_{c}$, two parents reproduce two offspring through one-point crossover (Lines 8 to 10). Then, mutation occurs on each offspring (Lines 11 to 16). It randomly alters a neuron-firing pattern with the mutation rate $P_{m}$. Binary tournament, crossover and mutation operators are executed repeatedly on $\mathcal{P}_{g}$ to reproduce $P$ offspring. The offspring $\left(\mathcal{O}_{g}\right)$ are combined with the parent population $\mathcal{P}_{g}$ to form $\mathcal{R}_{g}$ (Line 19 ).

Environmental selection follows reproduction. Best $P$ individuals are selected from $2 P$ individuals in $\mathcal{R}_{g}$ as the next generation population $\left(\mathcal{P}_{g+1}\right)$. First, the individuals in $\mathcal{R}_{g}$ are ranked based on the $\alpha$-dominance relationships among them. Non-dominated individuals are on the first rank. The $i$-th rank consists of the individuals dominated only by the individuals on the $(i-1)$-th rank. Ranked individuals are stored in $\mathcal{F}$ (Line 20). $\mathcal{F}_{i}$ contains the individuals at the $i$-th rank.

Then, the individuals in $\mathcal{F}$ move to $\mathcal{P}_{g+1}$ on a rank by rank basis, starting with $\mathcal{F}_{1}$ (Lines 23 to 26). If the number of individuals in $\mathcal{P}_{g+1} \cup \mathcal{F}_{i}$ is less than $P, \mathcal{F}_{i}$ moves to $\mathcal{P}_{g+1}$. Otherwise, a subset of $\mathcal{F}_{i}$ moves to $\mathcal{P}_{g+1}$. The subset is selected based on the crowding distance metric, which measures the distribution (or diversity) of individuals in the objective space [5] (Lines 27 and 28). The metric computes the distance between two closest neighbors of an individual in each objective and sums up the distances associated with all objectives. A higher crowding distance means that an individual in question is more distant from its neighboring individuals in the objective space. In Line 27 , the

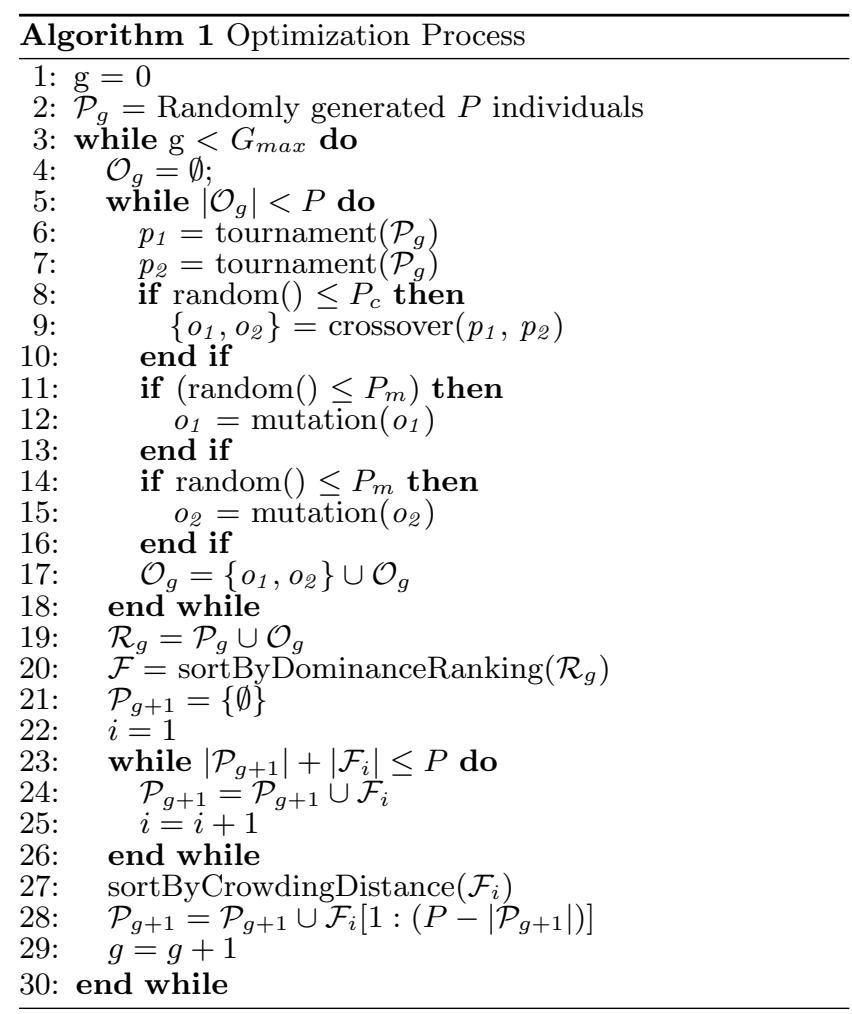

individuals in $\mathcal{F}_{i}$ are sorted in an descending order based on their crowding distance measures. The individuals with higher crowding distance have higher chances to be selected to $\mathcal{P}_{g+1}$ (Line 28 ).

\subsection{Simulation Evaluation}

This section evaluates the proposed EMOA through simulations. A simulated neuronal network is obtained with a two-step procedure. The first step utilizes NeuGen [7] to generate a network of $N$ neurons. The second step forms a tree structure with those neurons based on a randomized $L$-ary tree construction algorithm. This algorithm generates a rooted tree in which each neuron has no more than $\mathcal{N}\left(L,(L+1)^{2}\right)$ child neurons. $\mathcal{N}$ denotes a normal distribution. $L$ and $L+1$ are the mean and the standard deviation of the number of child neurons for each neuron. This paper uses a randomized 2-ary (i.e., binary) tree that contains 40 neurons and 10 sensors.

The proposed EMOA is configured with a set of parameters shown in Table 1. $Q$ denotes the total number of time slots in an individual $(Q=15$ in Fig. 8). Every simulation result is the average of the results from 20 independent simulations.

This simulation study considers noise that varies the time for a signal to travel through a neuron from $T_{u}$ to $T_{u}^{\prime}$ as follows.

$$
T_{u}^{\prime}=\beta\left(3 \times \mathcal{G}\left(k_{1}, \theta_{1}\right)+\mathcal{G}\left(k_{2}, \theta_{2}\right)\right) \times T_{u}
$$

$\mathcal{G}$ denotes a gamma distribution. This noise model is designed based on existing empirical and theoretical studies on time distributions for synapitic delay and refectory pe$\operatorname{riod}[2,10,15]$. This noise $\left(T_{u}^{\prime}-T_{u}\right)$ varies $t_{a}$ in Equations 1 and 2 and in turn determines $e_{L}$ and $e_{R}$ in Equations 3 and 4 . 
Table 1: Simulation Configurations

\begin{tabular}{|l|c|}
\hline Parameter & Value \\
\hline \hline \# of objective value samples per individual & 30 \\
Confidence level $(\alpha)$ & 0.95 \\
Total \# of generations $\left(G_{\max }\right.$ in Algo. 1$)$ & 200 \\
Population size $(P$ in Algo. 1$)$ & 100 \\
Crossover rate $\left(P_{c}\right.$ in Algo. 1$)$ & 0.9 \\
Mutation rate $\left(P_{m}\right.$ in Algo. 1$)$ & $1 / Q$ \\
Noise shape parameters $k_{1}$ and $k_{2}$ (Eq. 8$)$ & 6 and 7 \\
Noise scale parameters $\theta_{1}$ and $\theta_{2}$ (Eq. 8$)$ & 0.4 and 0.5 \\
Noise strength $\beta$ (Eq. 8$)$ & 0.1 \\
\hline
\end{tabular}

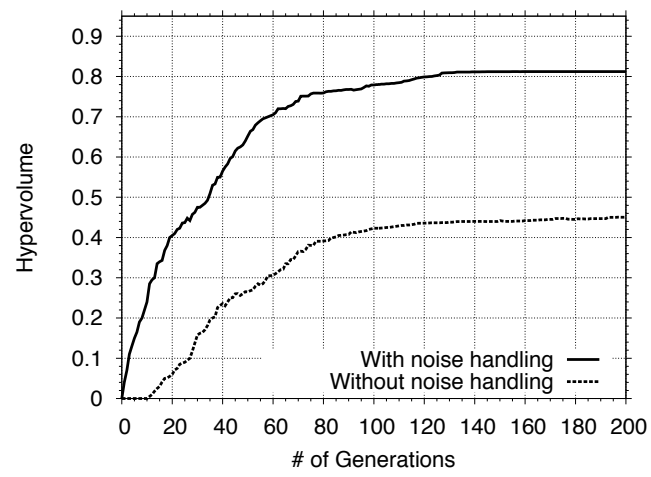

Figure 10: Hypervolume Measures with and without Noise Handling

Figure 10 illustrates how individuals increase the union of the hypervolumes that they dominate in the objective space as the number of generations grows. The hypervolume metric quantifies the optimality and diversity of individuals [32]. A higher hypervolume means that individuals are closer to the Pareto-optimal front and more diverse in the objective space. Figure 10 shows the hypervolume measures with and without the $\alpha$-dominance operator. When the $\alpha$-dominance operator is disabled, a classical dominance operator [23] is used in Algorithm 1. When the $\alpha$-dominance operator is enabled, hypervolume measure converges around the 130th generation. When it is disabled, the proposed EMOA's convergence speed and optimization results significantly degrade. The hypervolume measure in the last generation degrades $44 \%$ with the $\alpha$-dominance operator disabled. Figure 10 demonstrates that the $\alpha$-dominance operator works effectively and efficiently.

Table 2 shows the average and standard deviation of each objective value that is obtained at the last generation. Figure 11 shows the objective space that plots the individuals obtained at the last generation. Those individuals approximate the Pareto front (i.e., the optimal trade-off) between signaling latency and robustness. Given the distributions of individuals in objective value ranges, signaling protocol designers can examine how they can trade one objective for another and choose a particular individual as the best TDMA schedule according to their preferences, priorities and/or constraints. For example, Figure 11 demonstrates that robustness improves $42 \%$ as signaling latency increases 20 to 30 .

Table 3 depicts the hypervolume measures at the last generation where the $\alpha$-dominance operator and two other noise-aware dominance operators are used with Algorithm 1.
Table 2: Objective Values

\begin{tabular}{|c|c||c|c|}
\hline \multicolumn{2}{|c|}{} & Latency $\left(f_{L}^{e}\right)$ & Robustness $\left(f_{R}^{e}\right)$ \\
\hline \hline \multirow{2}{*}{ W/ Noise Handling } & Mean & 23.02 & 4.15 \\
\cline { 2 - 4 } & Var. & 6.77 & 0.56 \\
\hline \hline \multirow{2}{*}{ W/o Noise Handling } & Mean & 30.43 & 1.33 \\
\cline { 2 - 4 } & Var. & 8.12 & 0.78 \\
\hline
\end{tabular}

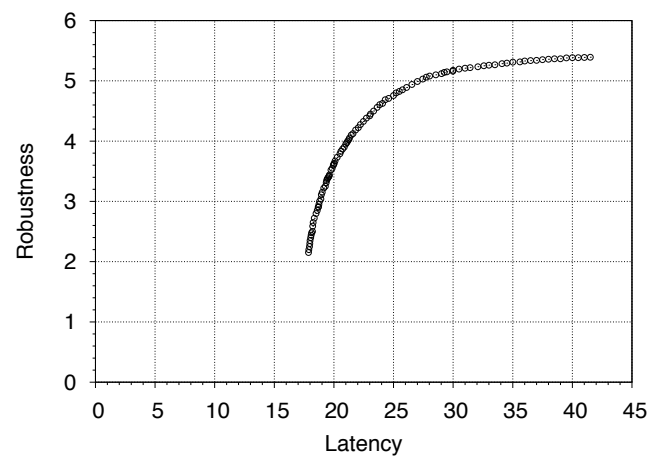

Figure 11: Latency-Robustness Tradeoff

- Mean-based dominance operator: It takes multiple samples, obtains mean values in different objectives and use them to rank individuals.

- Gaussian dominance operator: It assumes Gaussian noise in objective values in advance (c.f. Section 2) [8] and performs noise-aware ranking of individuals.

As shown in Table 3, the $\alpha$-dominance operator yields the highest hypervolume measure. It outperforms a mean-based dominance operator and a Gaussian dominance operator by $44 \%$ and $37 \%$. Through a single-tail $t$-test and a $U$-test, it is verified that the $\alpha$-dominance operator significantly outperforms the other two operators with the confidence level of $95 \%$.

\section{CONCLUSIONS}

This paper focuses on TDMA-based neuronal signaling and formulates a noisy multiobjective optimization problem that consists of signaling latency and robustness objectives. The problem contains environmental noise inherent in neuronal signaling. The proposed EMOA is designed to solve this problem with nonparametric (i.e.. distribution-free) statistical dominance operator. Simulation results show that the proposed EMOA handles noise well and obtains quality TDMA signaling schedules efficiently and effectively.

\section{REFERENCES}

[1] S. Balasubramaniam, N. T. Boyle, A. Della-Chiesa, F. Walsh, A. Mardinoglu, D. Botvich, and A. Prina-Mello. Development of artificial neuronal networks for molecular communication. Nano Communication Networks, 2(2-3), 2011.

[2] R. P. Betts, D. M. Johnston, and B. H. Brown. Nerve fibre velocity and refractory period distributions in nerve trunks. J. Neurol. Neurosurg. Psychiatry, 39(7):694-700, 1976.

[3] R. Cajal. Histology of nervous system of man and vertebrates. Oxford University Press, 1995. 
Table 3: Comparison among Different Noise-aware Dominance Operators with Hypervolume

\begin{tabular}{|c||c|c|c|}
\hline & Mean & Gaussian & $\alpha$-dominance \\
\hline \hline Hypervolume & 0.45 & 0.51 & 0.81 \\
\hline Difference & $44 \%$ & $37 \%$ & - \\
\hline
\end{tabular}

[4] D. J. Chew, L. Zhu, E. Delivopoulos, I. R. Minev, K. M. Musick, C. A. Mosse, M. Craggs, N. Donaldson, S. P. Lacour, S. B. McMahon, and J. W. Fawcett. A microchannel neuroprosthesis for bladder control after spinal cord injury in rat. Sci Transl Med 6 November, 5(210), 2013.

[5] K. Deb, S. Agrawal, A. Pratab, and T. Meyarivan. A fast elitist non-dominated sorting genetic algorithm for multi-objective optimization: NSGA-II. In Proc. Conf. Parallel Problem Solving from Nature, 2000.

[6] A. Destexhe. Neuronal noise. Springer, 2012.

[7] J. P. Eberhard, A. Wanner, and G. Wittum. NeuGen: A tool for the generation of realistic morphology of cortical neurons and neural networks in 3D. Neurocomputing, 70(1-3):327-342, 2006.

[8] H. Eskandari, C. D. Geiger, and R. Bird. Handling uncertainty in evolutionary multiobjective optimization: SPGA. In Proc. IEEE Congress on Evolutionary Computation, 2007.

[9] R. A. Freitas. Current status of nanomedicine and medical nanorobotics. J. Computational and Theoretical Nanoscience, 2(1):1-25, 2005.

[10] F. Gabbiani and S. J. Cox. Mathematics for Neuroscientists. Academic Press, 2010.

[11] N. Grossman, K. Nikolic, and P. Degenaar. The neurophotonic interface: stimulating neurons with light. The Neuromorphic Engineer, 2008.

[12] C. Hosokawa, Y. Sakamoto, S. N. Kudoh, Y. Hosokawa, and T. Taguchi. Femtosecond laser-induced stimulation of a single neuron in a neuronal network. Appl. Phys. A, 110(3), 2013.

[13] J. B. Hursh. Conduction velocity and diameter of nerve fibers. Amer. J. Physiol., 127, 1939.

[14] S. B. Jun, M. R. Hynd, N. Dowell-Mesfin, K. L. Smith, J. N. Turner, W. Shain, and S. J. Kima. Low-density neuronal networks cultured using patterned polyl-L-lysine on microelectrode arrays. Journal of Neurosci. Methods, 160(2), 2007.

[15] B. Katz and R. Miledi. The measurement of synaptic delay, and the time course of acetylcholine release at the neuromuscular junction. Proc. R. Soc. Lond. B. Biol. Sci., 161:483-495, 1965.

[16] F. Morin, N. Nishimura, L. Griscomb, B. LePioufle, H. Fujita, Y. Takamura, and E. Tamiya. Constraining the connectivity of neuronal networks cultured on microelectrode arrays with microSSuidic techniques: A step towards neuron-based functional chips. Biosensors and Bioelectronics, 21, 2006.

[17] T. Nakano, A. Eckford, and T. Haraguchi. Molecular Communication. Cambridge University Press, 2013.

[18] T. D. Nguyen-Vu, H. Chen, A. M. Cassell, R. J. Andrews, M. Meyyappan, and J. Li. Vertically aligned carbon nanofiber architecture as a multifunctional 3-D neural electrical interface. IEEE Trans. Biomed. Eng., 54(6), 2007.

[19] K. Partridge, B. Dahlquist, A. Veiseh, A. Cain, A. Foreman, J. Goldberg, and G. Borriello. Empirical measurements of intrabody communication performance under varied physical configurations. In Proc. User Interface Softw. Technol. Symp., 2001.

[20] S. Pautot, C. Wyart, and E. Y. Isacoff. Colloid-guided assembly of oriented 3D neuronal networks. Nature Methods, 5(8):735-740, 2008.

[21] P. E. M. Purnick and R. Weiss. The second wave of synthetic biology from modules to systems. Nature Review Molecular Cell Biology, 10:410-422, 2009.

[22] D. Shire, S. Kelly, J. Chen, P. Doyle, M. Gingerich, S. Cogan, W. Drohan, O. Mendoza, L. Theogarajan, J. Wyatt, and J. Rizzo. Development and implantation of a minimally invasive wireless subretinal neurostimulator. IEEE Trans. Biomedical Engineering, 56(10):2502-2511, 2009.

[23] N. Srinivas and K. Deb. Multiobjective function optimization using nondominated sorting genetic algorithms. Evol. Computat., 2(3), 1995.

[24] G. Stuart, J. Schiller, and B. Sakmann. Action potential initiation and propagation in rat neocortical pyramidal neurons. Journal of Physiology, 505.3, 1997.

[25] T. Suda, M. Moore, T. Nakano, R. Egashira, and A. Enomoto. Exploratory research on molecular communication between nanomachines. In Proc. ACM Genetic and Evol. Computat. Conference, 2005.

[26] J. Suzuki, S. Balasubramaniam, and A. Prina-Mello. Multiobjective TDMA optimization for neuron-based molecular communication. In Proc. of the 7th Int'l Conference on Body Area Networks. September 2012.

[27] J. Teich. Pareto-front exploration with uncertain objectives. In Proc. Int'l Conf. on Evol. Multi-Criterion Optimization, 2001.

[28] M. Wormington, C. Panaccione, K. M. Matney, and D. K. Bowen. Characterization of structures from x-ray scattering data using genetic algorithms. Phil. Trans. R. Soc. Lond. A, 357(1761), 1999.

[29] C. Wyart, C. Ybert, L. Bourdieu, C. Herr, C. Prinz, and D. Chatenay. Constrained synaptic connectivity in functional mammalian neuronal networks grown on patterned surfaces. J. Neurosci. Methods, 117(2), 2002.

[30] T. Yagi. Biohybrid visual prosthesis for restoring blindness. Int'l J. of Applied Biomedical Engineering, 2(1):1-5, 2009.

[31] T. Zimmerman. Personal area networks (PAN): Near-field intra-body communication. IBM Systems Journal, 35(3-4), 1996.

[32] E. Zitzler and L. Thiele. Multiobjective optimization using evolutionary algorithms: A comparative study. In Proc. Int'l Conf. on Parallel Problem Solving from Nature, 1998.

[33] E. Zitzler and L. Thiele. Multiobjective evolutionary algorithms: a comparative case study and the strength pareto approach. IEEE Trans. Evol. Computat., 3(4), 1999. 